\title{
Large linear magnetoelectric effect and field-induced ferromagnetism and ferroelectricity in $\mathrm{DyCrO}_{4}$
}

Xudong Shen ${ }^{1,2}$, Long Zhou ${ }^{1,2}$, Yisheng Chai ${ }^{1,3}$, Yan Wu ${ }^{4}$, Zhehong Liu ${ }^{1,2}$, Yunyu Yin ${ }^{1,2}$, Huibo Cao ${ }^{4}$, Clarina Dela Cruz ${ }^{4}$, Young Sun (1) ${ }^{1,2}$, Changqing Jin ${ }^{1,2}$, Angel Muñoz $z^{5}$ José Antonio Alonso $\mathbb{B}^{6}$ and Youwen Long ${ }^{1,2,7}$

\begin{abstract}
All the magnetoelectric properties of scheelite-type $\mathrm{DyCrO}_{4}$ are characterized by temperature- and fielddependent magnetization, specific heat, permittivity, electric polarization, and neutron diffraction measurements. Upon application of a magnetic field within $\pm 3 \mathrm{~T}$, the nonpolar collinear antiferromagnetic structure leads to a large linear magnetoelectric effect with a considerable coupling coefficient. An applied electric field can induce the converse linear magnetoelectric effect, realizing magnetic field control of ferroelectricity and electric field control of magnetism. Furthermore, a higher magnetic field (>3 T) can cause a metamagnetic transition from the initially collinear antiferromagnetic structure to a canted structure, generating a large ferromagnetic magnetization up to $7.0 \mu_{\mathrm{B}} \mathrm{f.u}^{-1}{ }^{-1}$. Moreover, the new spin structure can break the space inversion symmetry, yielding ferroelectric polarization, which leads to coupling of ferromagnetism and ferroelectricity with a large ferromagnetic

component.
\end{abstract}

\section{Introduction}

The linear magnetoelectric (ME) effect and multiferroicity enable control of polarization $P$ (magnetization $M$ ) by a magnetic (electric) field, which is beneficial for applications in spintronic devices, nonvolatile memories, high-sensitivity magnetic field sensors, etc ${ }^{1-5}$. In the linear ME effect, the induced electric polarization or magnetization is proportional to the applied magnetic field $H$ or electric field $E$, which can be expressed as $P=\alpha H$ or $\mu_{0} M$ $=\alpha E^{6,7}$, where $\alpha$ denotes the linear ME coefficient and $\mu_{0}$ denotes the magnetic permeability of a vacuum. Since the first observation of the linear ME effect in $\mathrm{Cr}_{2} \mathrm{O}_{3}$ in the $1960 \mathrm{~s}^{8,9}$, the search for single-phase ME materials with larger $\alpha$ values has attracted much attention. The

Correspondence: Youwen Long (ywlong@iphy.ac.cn)

${ }^{1}$ Beijing National Laboratory for Condensed Matter Physics, Institute of Physics, Chinese Academy of Sciences, Beijing 100190, China

${ }^{2}$ School of Physics, University of Chinese Academy of Sciences, Beijing 100049, China

Full list of author information is available at the end of the article. maximum $\alpha$ obtained for a nonpolar antiferromagnetic (AFM) structure is $<40 \mathrm{ps} \mathrm{m}^{-1}$ usually ${ }^{6}$. It is important to obtain a larger and constant ME effect in wider temperature and/or magnetic field regions for potential applications.

In the 1990s, the term "multiferroics" was proposed to describe materials that simultaneously exhibit more than one ferroic order, such as ferromagnetism, ferroelectricity, and ferroelasticity, in a single phase ${ }^{10}$. Among the different multiferroics, the spin-ordering-induced ferroelectrics (i.e., ME multiferroics) attracted significant attention owing to the strong ME coupling ${ }^{11-15}$. Based on the magnetic space group, collinear ferromagnetic (FM) spin alignment cannot break the spatial inversion symmetry. Therefore, electric polarization in single-phase ME multiferroics is often induced by some peculiar AFM or canted AFM spin structures instead of collinear ferromagnetism ${ }^{16}$. Consequently, the magnetic moment is too small to be effectively controlled ${ }^{17-19}$. It is very challenging to obtain a material with strongly coupled

\section{(c) The Author(s) 2019}

(c) (i) Open Access This article is licensed under a Creative Commons Attribution 4.0 International License, which permits use, sharing, adaptation, distribution and reproduction cc) in any medium or format, as long as you give appropriate credit to the original author(s) and the source, provide a link to the Creative Commons license, and indicate if changes were made. The images or other third party material in this article are included in the article's Creative Commons license, unless indicated otherwise in a credit line to the material. If material is not included in the article's Creative Commons license and your intended use is not permitted by statutory regulation or exceeds the permitted use, you will need to obtain permission directly from the copyright holder. To view a copy of this license, visit http://creativecommons.org/licenses/by/4.0/. 
FM-ferroelectric properties and a considerable magnetic moment.

$\mathrm{DyCrO}_{4}$ has a rare $\mathrm{Cr}^{5+}$ valence state. Under ambient conditions, it crystallizes into a zircon-type crystal structure with the space group of $I 4_{1} / a m d^{20,21}$. An FM phase transition with a large magnetocaloric effect occurs at approximately $23 \mathrm{~K}^{22-24}$. Moreover, the zircon-type structure is sensitive to external pressure. A pressureinduced irreversible structural phase transition toward a scheelite-type new phase with $I 4_{1} / a$ symmetry has already been reported ${ }^{25}$. In contrast to the FM zircon phase, the scheelite phase exhibits a long-range AFM transition ${ }^{26-28}$. In this study, scheelite-type $\mathrm{DyCrO}_{4}$ was prepared in a polycrystalline form by a high-pressure and moderatetemperature treatment method (see "Materials and methods" below). Based on the powder X-ray diffraction (XRD) and neutron diffraction results, there is no discernable impurity phase, such as the residual FM zircon phase, or decomposed $\mathrm{DyCrO}_{3}$ perovskite phase (see Supplementary Figs. S1 and S2 for details). This compound has a nonpolar collinear AFM ground state with zero magnetic field. Within $\mu_{0} H \approx \pm 3 \mathrm{~T}$, a large and almost constant linear ME effect is observed, enabling $H$ control of $P$ and $E$ control of $M$. Under higher magnetic fields, a metamagnetic transition occurs and is accompanied by a large FM magnetization. Furthermore, the new spin structure can break the spatial inversion symmetry and thereby generate ferroelectric polarization. The presented $\mathrm{DyCrO}_{4}$ paves the way for novel investigations on simultaneous direct and converse linear ME effects and FM-ferroelectrics with large magnetic moments.

\section{Materials and methods}

\section{Sample synthesis and XRD}

To fabricate the scheelite-type $\mathrm{DyCrO}_{4}$ sample, the zircon-type sample was prepared at ambient pressure as a precursor, as reported in a previous study ${ }^{26}$. The prepared zircon-type $\mathrm{DyCrO}_{4}$ powders were pressed into a golden cylinder with a diameter of $4.0 \mathrm{~mm}$ and height of $3.0 \mathrm{~mm}$. The cylinder was treated at 6-8 GPa and $700-750 \mathrm{~K}$ for 10-30 min on a cubic-anvil-type high-pressure apparatus using pyrophyllite as a pressure transmission medium. When the heating power was turned off, the pressure was slowly released. The crystal quality and structure were analyzed by powder XRD using a Huber diffractometer with $\mathrm{Cu} \mathrm{K} \mathrm{K}_{\alpha 1}$ radiation at $40 \mathrm{kV}$ and $30 \mathrm{~mA}$. The XRD data were acquired at room temperature in the $2 \theta$ range of $10^{\circ}-100^{\circ}$ with steps of $0.005^{\circ}$ and analyzed by Rietveld refinement using the GSAS software ${ }^{29}$.

\section{Magnetic, specific heat, dielectric, and pyroelectric current measurements}

The magnetic susceptibility $(\chi)$ and magnetization $(M)$ were measured using a magnetic property measurement system (Quantum Design, MPMS-3). Both zero fieldcooling (FC) and FC modes were employed for $\chi$ measurements at $0.01 \mathrm{~T}$. The field-dependent $M$ measurements were performed at different temperatures. The specific heat $\left(C_{\mathrm{p}}\right)$ and electrical properties were measured using a physical property measurement system (Quantum Design, PPMS-9T). The $C_{\mathrm{p}}$ data were acquired during cooling in the range of $50-2 \mathrm{~K}$ with different magnetic fields. For the measurements of the relative dielectric constant $\varepsilon_{\mathrm{r}}$, dielectric loss $\tan \delta$, and pyroelectric current density $(\delta)$, the specimens were fabricated as thin plates with a typical surface area of $1-2 \mathrm{~mm}^{2}$ and a thickness of $0.2 \mathrm{~mm}$. Silver electrodes were coated onto the two surfaces of the plate for electrical measurements. Different frequencies of $1 \mathrm{kHz}, 10 \mathrm{kHz}, 0.1 \mathrm{MHz}$, and $1 \mathrm{MHz}$ were applied for the dielectric constant and dielectric loss measurements at selected magnetic fields in the range of 0-9 $\mathrm{T}$ with intervals of $1 \mathrm{~T}$ using an Agilent E4980A Precision LCR Meter while sweeping the temperature from 2 to $50 \mathrm{~K}$ with a heating rate of $2 \mathrm{~K} \mathrm{~min}^{-1}$. The pyroelectric current was measured by a Keithley 6517B precise electrometer while sweeping the temperature from 2 to $50 \mathrm{~K}$ at a rate of $2 \mathrm{~K} \mathrm{~min}^{-1}$. Before this measurement, the sample was poled from 50 to $2 \mathrm{~K}$ with a poling electric field of $E_{\mathrm{pol}}= \pm 1.08 \mathrm{MV} \mathrm{m}^{-1}$. Once the temperature was decreased to $2 \mathrm{~K}, E_{\mathrm{pol}}$ was switched off and $J$ was measured as described above. The magneticfield-dependent current was measured while sweeping the magnetic field at a rate of $0.01 \mathrm{~T} \mathrm{~s}^{-1}$. In these measurements, the PPMS was used to provide low-temperature and magnetic field environments. The electric polarization $(P)$ was obtained by integrating $J$ as a function of time. In addition, we measured the electric-field-induced magnetization using MPMS-3 with different electric fields produced by the Keithley 6517B electrometer. Before the measurement, the sample was cooled from 50 to $10 \mathrm{~K}$ at a rate of $2 \mathrm{~K} \mathrm{~min}^{-1}$ at $E_{\mathrm{pol}}=0.5 \mathrm{MV} \mathrm{m}^{-1}$ and $\mu_{0} H=4 \mathrm{~T}$. Once the temperature was decreased to $10 \mathrm{~K}$, both $E_{\mathrm{pol}}$ and $H$ were switched off, and then $M$ was measured during heating from 10 to $26.5 \mathrm{~K}$ at fixed temperatures and selected electric fields.

\section{Neutron diffraction measurements}

The neutron powder diffraction (NPD) measurements were carried out using the constant-wavelength highresolution neutron powder diffractometer HB2A at Oak Ridge National Laboratory (ORNL). The temperature was controlled by a Lakeshore Bridge, while the magnetic field was generated by an Oxford- $5 \mathrm{~T}$ superconducting magnet. The temperature-dependent diffraction profiles were measured at a wavelength of $\lambda=2.41 \AA$ with the collimation out-high intensity mode. The overall powder mass was $1.3 \mathrm{~g}$. To reduce the thickness to enable a neutron transmission rate of approximately $90 \%$, the sample 
powders were spread out inside a $30 \times 48 \times 0.25 \mathrm{~mm}^{3}$ sample holder; their thickness was approximately $0.15 \mathrm{~mm}$. The empty space in the sample holder was filled with Fluorinert to help fix the powders under a low temperature. The data were measured at different magnetic fields of $0,1,3$, and $4.3 \mathrm{~T}$. The magnetic refinements were carried out by the Rietveld method with the FullProf $\operatorname{program}^{30}$.

\section{Results and discussion}

As shown in Fig. 1a, the scheelite-type $\mathrm{DyCrO}_{4}$ with a space group of $I 4_{1} / a$ consists of spatially isolated $\mathrm{CrO}_{4}$ tetrahedra and edge-sharing $\mathrm{DyO}_{8}$ dimers ${ }^{20,26}$. According to the space group, $\mathrm{DyCrO}_{4}$ is centrosymmetric without spontaneous ferroelectric polarization in the crystal structure. With the decrease in temperature to $T_{\mathrm{N}}=24 \mathrm{~K}$, both the magnetic susceptibility measured at $0.01 \mathrm{~T}$ and specific heat at zero field show an AFM phase transition (Fig. 1b). Moreover, the NPD results at zero field (Fig. S2) suggest a collinear AFM spin structure composed of $\mathrm{Dy}^{3+}$ and $\mathrm{Cr}^{5+}$ spin sublattices, as presented in Fig. 1a. The magnetic symmetry analysis illustrates that the collinear AFM structure of $\mathrm{DyCrO}_{4}$ has a magnetic point group of $2^{\prime} / m$, where the two-fold rotation is along the $c$ axis, whereas the mirror is perpendicular to the $c$ axis. This magnetic point group gives the ME tensor $\alpha_{\mathrm{ME}}$ with the form shown in Eq. (1). Therefore, although the magnetic group is nonpolar, it allows nonzero linear ME components, such as $\alpha_{13}\left(\alpha_{31}\right)$ and $\alpha_{23}\left(\alpha_{32}\right)$. This implies that an external $H$ parallel to the $c$ axis induces $P$ along the $a / b$ axis or vice versa while maintaining the initial spin structure, which can in principle lead to a linear ME effect.

$$
\alpha_{M E}=\left(\begin{array}{ccc}
0 & 0 & \alpha_{13} \\
0 & 0 & \alpha_{23} \\
\alpha_{31} & \alpha_{32} & 0
\end{array}\right)
$$

To demonstrate the linear ME effect in an experiment, we first measure the temperature dependences of the relative dielectric permittivity and dielectric loss at different magnetic fields with an $H \perp E$ configuration, as shown in Fig. 1c, d. At $\mu_{0} H=0 \mathrm{~T}$, no anomaly in permittivity is observed in the measured temperature range. In contrast, a moderate magnetic field can induce an apparent dielectric peak near $T_{\mathrm{N}}$. Moreover, with the increase in the magnetic field, the dielectric peak becomes sharper and gradually shifts toward lower temperatures (Fig. 1c). On the other hand, at a fixed $H$, the dielectric peak is independent of the measurement frequency (Fig. S3). Analogous phenomena are observed in the dielectric loss with a maximum value $<0.032$ (Fig. 1d), indicating the intrinsic dielectric variations with the magnetic field. The dielectric measurements thus suggest $H$-induced $P$.

To probe the off-diagonal nonzero components of the ME tensor $\alpha_{\mathrm{ME}}$ of the polycrystalline sample, we employ an $\mathrm{ME}$ annealing procedure with an $H \perp E$ configuration, as described in the "Materials and methods" section and reported in the literature ${ }^{31,32}$. In a polycrystalline sample with a random distribution of the grain orientation, such an ME annealing procedure can be used to choose one of the ME domains for each grain so that the macroscopic

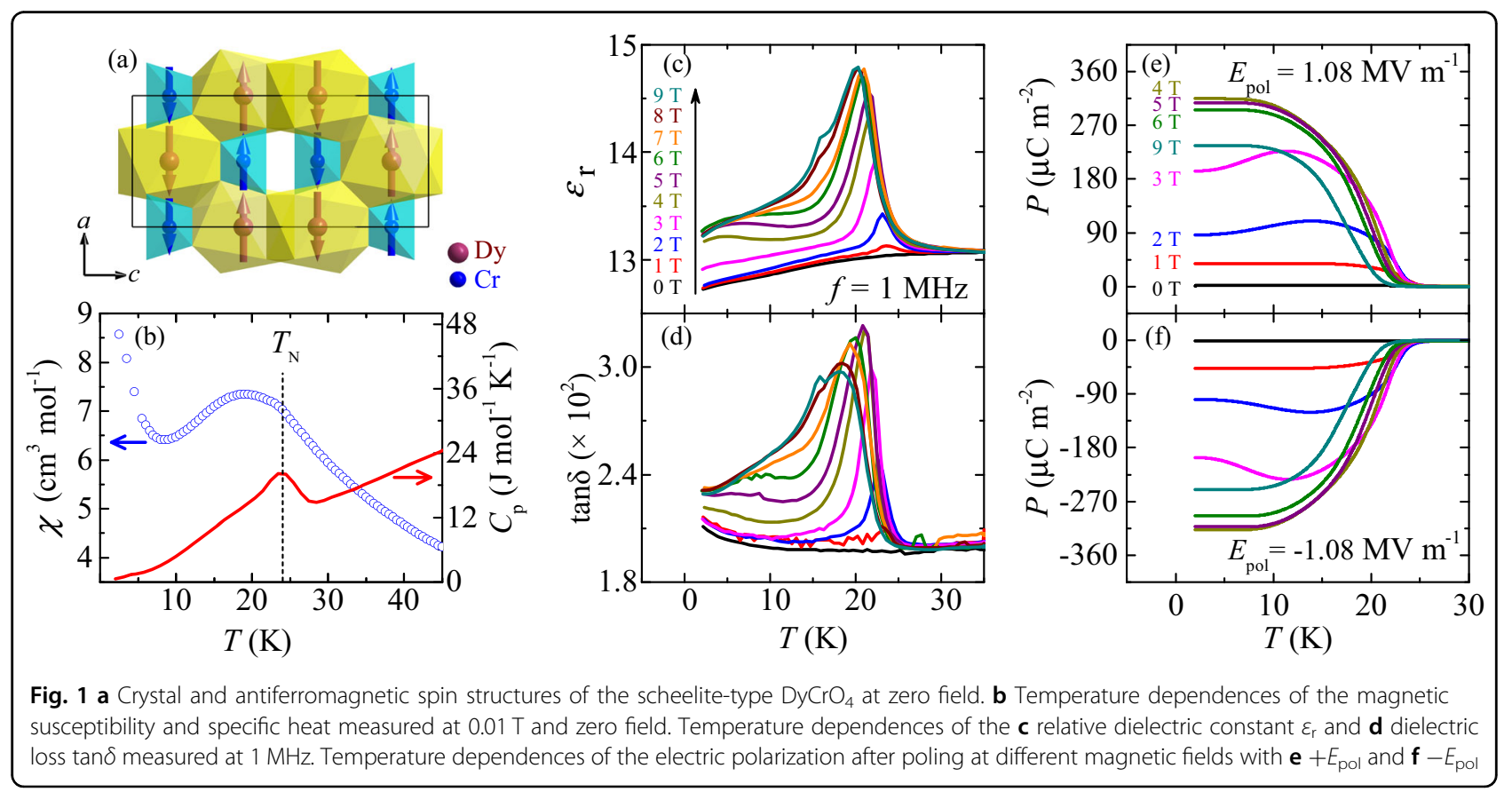



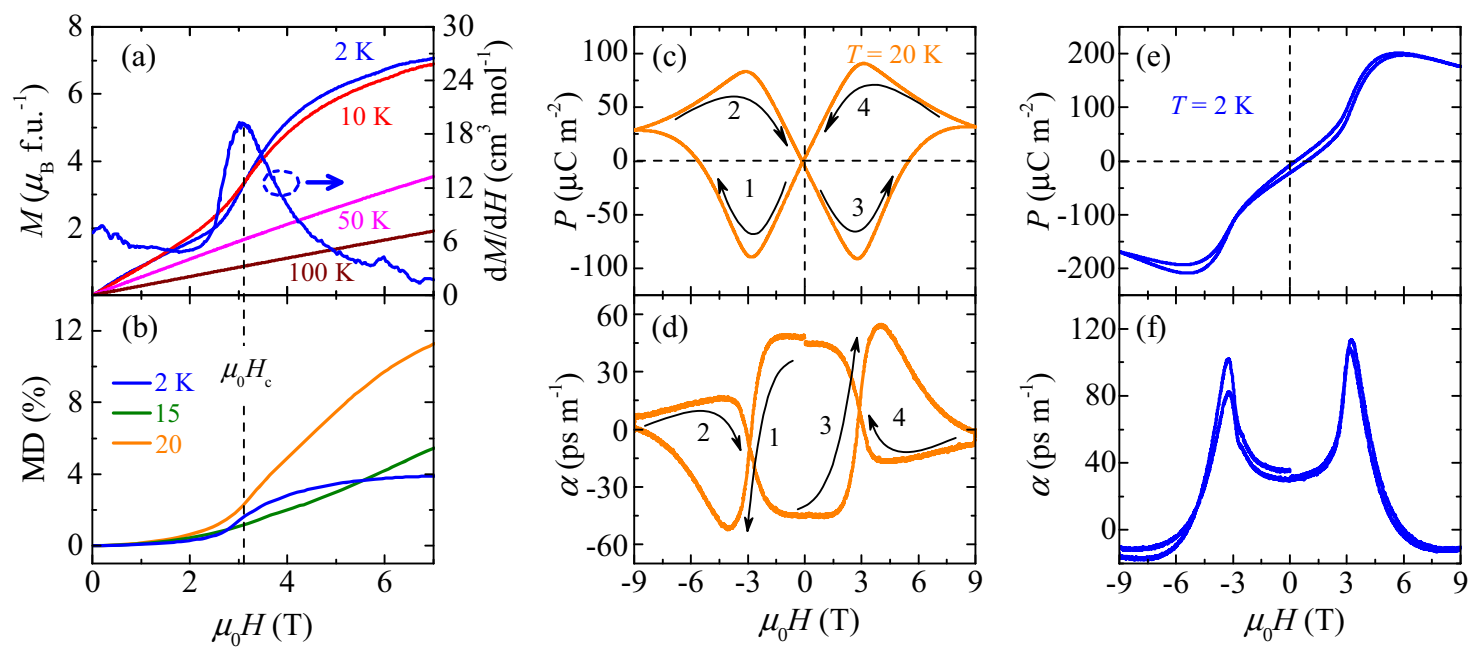

Fig. 2 a Magnetization of the scheelite-type $\mathrm{DyCrO}_{4}$ and its derivative as a function of the applied magnetic field. $\mathbf{b}$ Magnetodielectric ratio $(\mathrm{MD})=$ $100 \% \times\left[\varepsilon\left(\mu_{0} H\right)-\varepsilon(0)\right] / \varepsilon(0)$ measured at different temperatures as a function of the magnetic field. Magnetic field dependences of the polarization and magnetoelectric coefficients $a$ at (c, d) 20 and (e, f) $2 \mathrm{~K}$

ME effect can be observed considering the symmetry requirement. The temperature-dependent pyroelectric current was measured at the selected magnetic fields (Fig. S4), and then the related polarization was obtained by integrating the pyroelectric current as a function of the time. Using this approach, no significant $P$ is observed at $\mu_{0} H=0 \mathrm{~T}$ at temperatures down to $2 \mathrm{~K}$, as shown in Fig. $1 \mathrm{e}$. However, under an external $H \perp E$, electric polarization is induced. The onset temperature of the induced $P$ is in close agreement with the position of the dielectric peak. Moreover, the sign of $H$-induced $P$ can be reversed by reversing the poling electric field $E_{\mathrm{pol}}$ while maintaining the magnitude of $P$, as shown in Fig. $1 \mathrm{f}$ and Fig. S4. These results confirm $H$-induced polarization in accordance with the nonzero off-diagonal components in $\alpha_{\mathrm{ME}}$, which is expected from the AFM spin structure of $\mathrm{DyCrO}_{4}$. Compared with the $H \perp E$ configuration, the induced $P$ observed in our sample with an $H / / E$ configuration is considerably reduced (Fig. S5), consistent with the null value of the diagonal components of the ME tensor as presented in Eq. (1), although the detected $P$ is not exactly zero due to the polycrystalline nature of the sample.

As shown in Fig. 1e, f, at $\mu_{0} H \leq 3 \mathrm{~T}$, the magnitude of $H$ induced $P$ increases with $H$ and exhibits a broad maximum at approximately $15 \mathrm{~K}$ and then slightly decreases at lower temperatures. Moreover, if one looks at the pyroelectric current as presented in Fig. S4, the sign of the current measured at 2 and $3 \mathrm{~T}$ changes to be negative below approximately $15 \mathrm{~K}$. This observation suggests that the $\mathrm{Cr}$ and Dy ions may have opposite contributions to the induced electric polarization $<3 \mathrm{~T}$. However, for $\mu_{0} H \geq 4 \mathrm{~T}$, the sign of the pyroelectric current is always positive. Correspondingly, the polarization gradually saturates and does not vary with further cooling. The essentially different polarization behaviors at lower and higher fields mentioned above are related to different spin structures due to a field-induced metamagnetic transition, as shown later. The maximum $P$ is observed in the range of 4-5 T, which may be attributed to the interaction between the Dy- $4 f$ and Cr- $3 d$ moments. Owing to the remarkable magnetic anisotropy, the applied magnetic field can change the magnetic state of Dy and thus affect the electric polarization. To elucidate the distinct polarization behaviors of these, we measured the magnetization as a function of the magnetic field at selected temperatures, as presented in Fig. 2a. Above $T_{\mathrm{N}}, M$ exhibits a linear relationship with $H$ owing to paramagnetism. Below $T_{\mathrm{N}}$, the linear magnetization behavior is also observed at lower fields owing to the collinear AFM ground state (see Fig. S6). However, with the increase in $H$ above a critical value $\left(H_{\mathrm{c}}\right)$, which is determined by the derivative of $M$ with respect to $H$, a metamagnetic transition occurs from the initial collinear AFM structure to a canted one. For example, at $2 \mathrm{~K}$, the critical field is $3.1 \mathrm{~T}$; a large magnetic moment up to $7.0 \mu_{\mathrm{B}} \mathrm{f}_{\mathrm{fu}}{ }^{-1}$ caused by the metamagnetic transition is observed at field up to $7 \mathrm{~T}$.

Further, the magnetic field-dependent dielectric constants were measured at selected temperatures (Fig. S7) to characterize the magnetodielectric (MD) effect (Fig. 2b). The MD curves display significant increases around $\mu_{0} H_{\mathrm{c}}$ due to the metamagnetic transition, suggesting a different polarization behavior between the cases below and above $\mu_{0} H_{\mathrm{c}}$. Moreover, the MD value at $20 \mathrm{~K}$ and $7 \mathrm{~T}$ reaches $11.4 \%$, which is larger than the maximum MD value for the single-crystal multiferroic $\mathrm{TbMnO}_{3}{ }^{11}$. The magneticfield-dependent polarization was also measured to 
distinguish the different ME features in $\mathrm{DyCrO}_{4}$. Figure 2c, e show the resulting $P$ calculated from the corresponding ME current measurement results (Fig. S8) at 20 and $2 \mathrm{~K}$, respectively. At $20 \mathrm{~K}$, the polarization variation is close to linear within $\pm \mu_{0} H_{\mathrm{c}}$ reflecting the linear ME effect related to the collinear AFM; a sharp variation occurred around the critical field, above which the linear ME behavior was affected. The calculated linear ME coefficient $\alpha$ of $\mathrm{DyCrO}_{4}$ at $20 \mathrm{~K}$ was approximately constant at $50 \mathrm{ps} \mathrm{m}^{-1}$ in the region within $\pm \mu_{0} H_{\mathrm{c}}$ (Fig. 2d). Usually, the linear ME effects observed in collinear AFM materials without spontaneous electric polarization are quite small. For example, for a $\mathrm{Cr}_{2} \mathrm{O}_{3}$ single crystal, which is a prototype system for studies on the linear ME effect, the value of $\alpha$ is only approximately $2-4 \mathrm{ps} \mathrm{m}^{-133,34}$. $\mathrm{Co}_{4} \mathrm{Nb}_{2} \mathrm{O}_{9}{ }^{35}$ and $\mathrm{LiCoPO}_{4}{ }^{36}$ exhibit larger linear $\mathrm{ME}$ effects, but the coupling coefficients are approximately 20-30 $\mathrm{ps} \mathrm{m}^{-1}$. Compared with those of most single-phase compounds with nonpolar collinear AFM spin structures, the $\alpha$ value for $\mathrm{DyCrO}_{4}$ is one of the highest reported, even though our sample is polycrystalline. The $\mathrm{TbPO}_{4}$ single crystal is an extraordinary example with an $\alpha$ value up to $730 \mathrm{ps} \mathrm{m}^{-1}$ but only below a lower AFM transition temperature of $2.38 \mathrm{~K}^{7}$. It is worth noting that a few ME multiferroics exhibit very large ME effects ${ }^{19,37-41}$. However, they often only occur near the phase transition boundaries, while away from them, the ME effects are considerably reduced. In contrast, the ME coefficient of $\mathrm{DyCrO}_{4}$ is relatively constant over the wide magnetic field range of $-\mu_{0} H_{\mathrm{c}}$ to $+\mu_{0} H_{\mathrm{c}}$. At low temperatures (e.g., $2 \mathrm{~K}$ ) (Fig. 2e, f), the linear ME behavior of $\mathrm{DyCrO}_{4}$ is also observed. At this temperature, the maximum $\alpha$ is increased to approximately $110 \mathrm{ps} \mathrm{m}^{-1}$ at $\mu_{0} H_{\mathrm{c}}$. We will later discuss more about the different $P-H$ behaviors observed at 20 and $2 \mathrm{~K}$.

In addition, we investigate the electric field-induced magnetization $M_{\mathrm{E}}$, i.e., the so-called converse ME effect ${ }^{2}$. Figure 3a shows the temperature dependence of $M_{\mathrm{E}}$ measured in different electric fields. Below $T_{\mathrm{N}}$, a considerable $E$-induced magnetization with magnitude tunable by the electric field is observed. Moreover, the sign of $M_{\mathrm{E}}$ can be reversed upon application of an opposite measurement electric field. These features are very similar to those of the $H$-induced $P$, as shown in Fig. 1e, f. To further analyze the linear dependence of $M_{\mathrm{E}}$ on the applied $E$, periodically varied electric fields (top panel in Fig. $3 \mathrm{~b}$ ) were used for $M_{\mathrm{E}}$ measurements at $10 \mathrm{~K}$ and zero magnetic field. The middle and bottom panels in Fig. 3b present the obtained values of $M_{\mathrm{E}}$ after ME cooling at $\mu_{0} H=4 \mathrm{~T}$ and $E_{\mathrm{pol}}=0.5$ and $-0.5 \mathrm{MV} \mathrm{m}^{-1}$. The magnitude of $M_{\mathrm{E}}$ periodically changes according to the periodic variation in the electric field. Furthermore, a linear relationship between $M_{\mathrm{E}}$ and applied $E$ is observed, as demonstrated in the inset of Fig. 3b, confirming the

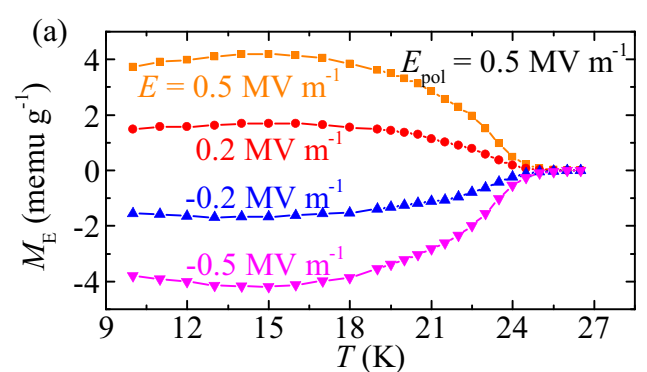

(b)

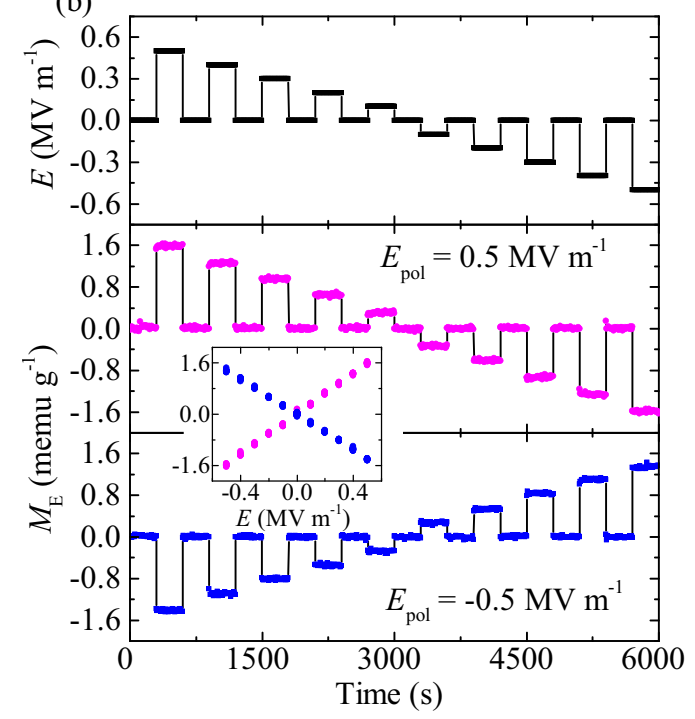

Fig. 3 a Temperature dependences of the induced magnetization magnetoelectric at selected electric fields for the scheelite-type DyCrO${ }_{4}$. b Prompt response of the magnetization to the regularly modulated electric field at $10 \mathrm{~K}$. The top panel shows the modulated electric field, while the middle and bottom panels show the magnetizations polarized at 4T with $E=0.5 \mathrm{MV} \mathrm{m}^{-1}$ and $E=-0.5 \mathrm{MV}$ $\mathrm{m}^{-1}$, respectively. $M$ as a linear function of $E$ is shown in the inset

converse linear ME effect of $\mathrm{DyCrO}_{4}$. The converse ME coefficient derived from $\mathrm{d} M_{\mathrm{E}} / \mathrm{d} E$ at $10 \mathrm{~K}$ is $26 \mathrm{ps} \mathrm{m}^{-1}$, which is slightly smaller than the value obtained from the $H$-induced $P$ at $20 \mathrm{~K}$.

The spin structure was studied in detail by NPD to investigate the spin origin of the electric polarization (see Supplementary Information for detailed analysis of NPD data). Figure 4a shows the net spin diffraction patterns obtained at $2 \mathrm{~K}$ under different magnetic fields after subtraction of the nuclear contribution from the collected NPD patterns (Fig. S2). At zero magnetic field, the spin structure composed of $\mathrm{Cr}^{5+}$ and $\mathrm{Dy}^{3+}$ was determined to be $k=(0,0,0)$ collinear AFM with spin moments parallel to the $a$ axis of the crystal lattice (Fig. 1a), agreeing well with a previous study ${ }^{28}$. The refined magnetic moments of $\mathrm{Cr}^{5+}$ and $\mathrm{Dy}^{3+}$ at $2 \mathrm{~K}$ and $0 \mathrm{~T}$ are $1.0 \pm 0.1$ and $9.6 \pm$ $0.5 \mu_{\mathrm{B}}$, respectively, as expected from the spin-only theoretical value for a $3 d-\mathrm{Cr}^{5+}$ ion $\left(1.0 \mu_{\mathrm{B}}\right)$ and obtained 

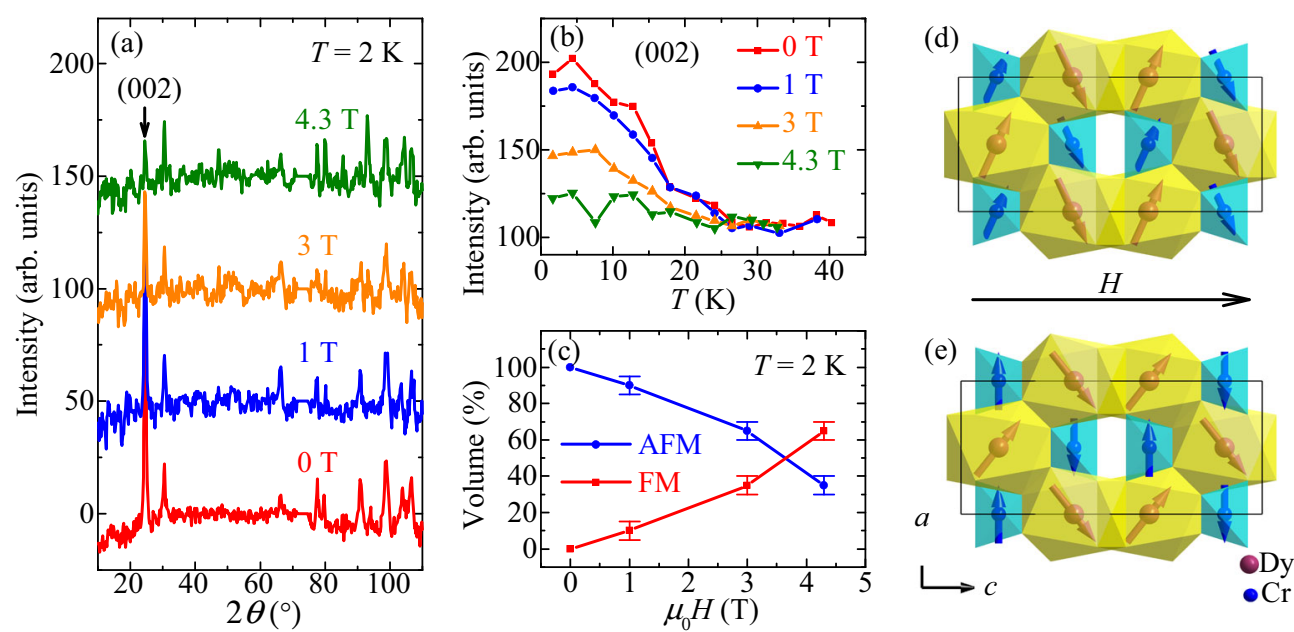

Fig. 4 a Net spin diffraction patterns obtained at $2 \mathrm{~K}$ and different magnetic fields after subtraction of the nuclear contribution for the scheelite-type Dy $\mathrm{CrO}_{4}$. $\mathbf{b}$ Temperature dependences of the magnetic (002) peak at different magnetic fields. $\mathbf{c}$ Magnetic field dependence of the phase composition at $\mathrm{T}=2 \mathrm{~K}$. Schematic spin structures including the ferromagnetic components of $\mathbf{d}$ both $\mathrm{Cr}^{5+}$ and $\mathrm{Dy}^{3+}$ spin sublattices and $\mathbf{e}$ only Dy ${ }^{3+}$ with $\mathrm{H}$ along the $c$ axis

considering the orbital contribution for a $4 f$-Dy ${ }^{3+}$ ion $\left(10.0 \mu_{\mathrm{B}}\right)$. According to the symmetry analysis, the collinear AFM structure of $\mathrm{DyCrO}_{4}$ has a nonpolar magnetic point group of $2^{\prime} / m$. The ME tensor of this magnetic group, as shown in Eq. (1), allows the generation of an intrinsic linear ME effect. Since both $\mathrm{Cr}^{5+}$ and $\mathrm{Dy}^{3+}$ take part in the AFM ordering and only a single anomaly is observed in magnetic susceptibility and specific heat curves (see Fig. $1 \mathrm{~b}$ and Fig. S9), the $\mathrm{Cr}^{5+}$ and $\mathrm{Dy}^{3+}$ ions should order simultaneously at $T_{\mathrm{N}}=24 \mathrm{~K}$. We thus conclude that these two types of ions contribute to the large linear $\mathrm{ME}$ effect in $\mathrm{DyCrO}_{4}$.

Upon the application of a magnetic field up to $4.3 \mathrm{~T}$ $\left(>\mu_{0} H_{\mathrm{c}}\right)$, the propagation vector remains unchanged $k=$ $(0,0,0)$. The most notable variation is that the intensity of the magnetic (002) reflection gradually decreases with the increase in $H$ (Fig. 4a, b and Fig. S10). This change implies that the magnetic moments rotate toward the applied magnetic field, inducing canting of the collinear AFM structure, which yields a net FM moment. Considering the polycrystalline nature of the sample, it is difficult to exactly fit the magnetic peaks under a magnetic field, owing to the variable orientation of the magnetic field with respect to different magnetic domains. Constraining our analysis to the two existing magnetic states (i.e., collinear AFM structure determined at zero field and collinear FM structure with all of the moments parallel to a fixed axis such as the $c$ axis), we can obtain the ratio between these two phases by fitting the scale factor while keeping the spin moments of $\mathrm{Cr}$ and Dy constant, as determined at $0 \mathrm{~T}$. The results evaluated at $2 \mathrm{~K}$ are shown in Fig. 4c. The composition of the FM phase at $4.3 \mathrm{~T}$ is approximately $65 \pm 5 \%$, indicating the strong ferromagnetism originating from the canted AFM alignment. After the metamagnetic phase transition above the critical magnetic field, the canted spin structure can be composed of both $\mathrm{Dy}^{3+}$ and $\mathrm{Cr}^{5+}$ spin sublattices (Fig. 4d) or just the dominant $\mathrm{Dy}^{3+}$ sublattice (Fig. 4e). In these two cases, the magnetic point group always changes from the initially nonpolar $2^{\prime} / m$ to a polar $m$. This polar point group can break the space inversion symmetry and thereby cause spontaneous ferroelectric polarization, yielding field-induced ferromagnetism and ferroelectricity in the compound with strong coupling with each other. It is worth noting that magnetostriction may occur under a high field, but this effect on the lattice change should be very small ${ }^{42}$. Based on the above ME results, the presented $\mathrm{DyCrO}_{4}$ is a rare material that simultaneously exhibits large direct and converse linear ME effects as well as fieldinduced ferromagnetism-ferroelectricity with a giant magnetic moment, making it distinct from other reported materials. For example, in the well-known type-II multiferroics such as $\mathrm{TbMnO}_{3}{ }^{11}$, the polarization originates from the spiral AFM ordering, and thus this structure does not exhibit a considerable net magnetic moment. Moreover, the converse ME effect is not observed in this compound because the magnetic field can easily change the spin structure. In compounds with (weak) ferromagnetism and electric polarization such as $\mathrm{EuTiO}_{3}$ and $\mathrm{GdFeO}_{3}$, although the polarization appears at a higher temperature (approximately $250 \mathrm{~K}$ in the $\mathrm{EuTiO}_{3}$ thin film), the FM transition occurs at a significantly lower temperature $\left(4.2 \mathrm{~K}\right.$ in the $\mathrm{EuTiO}_{3}$ thin film), indicating negligible ME coupling ${ }^{43}$. In contrast to the field-induced polarization, in the weakly $\mathrm{FM} \mathrm{GdFeO}_{3}$, the magnetic field rapidly suppresses the polarization to zero at $2-3 \mathrm{~T}^{44}$. 


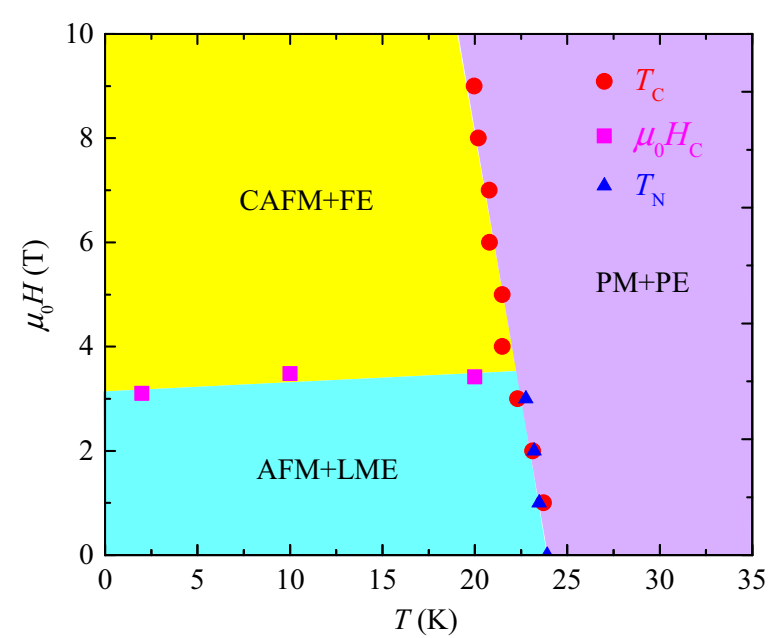

Fig. 5 Magnetic and electrical phase diagram of the scheelite-type DyCrO 4 . PM paramagnetic, PE paraelectric, FE ferroelectric, LME linear magnetoelectric, CAFM canted antiferromagnetic

$\mathrm{DyFeO}_{3}$ also has a field-induced ferroelectric state, but the accompanying magnetic moment is limited $\left(<1.0 \mu_{\mathrm{B}}\right.$ f.u. $\left.{ }^{-1}\right)^{45}$.

Using the experimental data, a characteristic magnetic and electric phase diagram can be obtained for different temperatures and magnetic fields. As shown in Fig. 5, the solid circles $\left(T_{\mathrm{c}}\right)$ and triangles $\left(T_{\mathrm{N}}\right)$ represent the phase boundary between the spin/electric order and disorder for scheelite-type $\mathrm{DyCrO}_{4}$. The values of $T_{\mathrm{c}}$ are determined from the dielectric peaks shown in Fig. 1c, while $T_{\mathrm{N}}$ is determined from specific heat data (Fig. S9). Below $T_{\mathrm{N}}$ and $\mu_{0} H_{\mathrm{c}}$, the compound has a nonpolar collinear AFM structure with a large linear ME effect. The $\alpha$ value of this phase is among the highest values for the single-phase ME materials with nonpolar collinear AFM structures. Moreover, the magnitude of $\alpha$ is almost constant in the wider magnetic field region of $-\mu_{0} H_{\mathrm{c}}$ to $+\mu_{0} H_{\mathrm{c}}$. In addition, the converse linear ME effect is also observed in this magnetic field region. Furthermore, the metamagnetic phase transition changes the nonpolar AFM spin structure to the polar structure with a large FM magnetization. A strong ferromagnetism coupled with ferroelectricity thus develops below $T_{\mathrm{c}}$ and above $\mu_{0} H_{\mathrm{c}}$. Note that, when the magnetic field increases to $9 \mathrm{~T}$, the compound is located near the paramagnetic and paraelectric phases at $20 \mathrm{~K}$, while the FM and ferroelectric phases set in at $2 \mathrm{~K}$. This difference is the reason why $H$-induced $P$ at 20 and $2 \mathrm{~K}$ displays different behaviors in high magnetic fields, as shown in Fig. 2c, e. The presented $\mathrm{DyCrO}_{4}$ is a rare material, which simultaneously displays large direct and converse linear ME effects in the nonpolar collinear AFM phase and field-induced ferromagnetism and ferroelectricity with a large magnetic moment.

\section{Acknowledgements}

This work was supported by the National Key R\&D Program of China (Grant Nos. 2018YFE0103200, 2018YFA0305700), the National Natural Science Foundation of China (Grant Nos. 11574378, 51772324, 11674384), the Chinese Academy of Sciences (Grant Nos. YZ201555, QYZDB-SSW-SLH013, GJHZ1773), and Spanish MINECO (Grant MAT2013-41099-R). Research conducted at ORNL's High Flux Isotope Reactor was sponsored by the Scientific User Facilities Division, Office of Basic Energy Sciences, US Department of Energy.

\section{Author details}

'Beijing National Laboratory for Condensed Matter Physics, Institute of Physics, Chinese Academy of Sciences, Beijing 100190, China. ${ }^{2}$ School of Physics, University of Chinese Academy of Sciences, Beijing 100049, China. ${ }^{3}$ Department of Applied Physics, Chongqing University, Chongqing 400044, China. ${ }^{4}$ Neutron Scattering Division, Neutron Scattering Science Directorate, Oak Ridge National Laboratory, Oak Ridge, TN 37831, USA. ${ }^{5}$ Universidad Carlos III, Avenida Universidad 30, 28911 Leganés-Madrid, Spain. ${ }^{6}$ Instituto de Ciencia de Materiales de Madrid, C.S.I.C., Cantoblanco, 28049 Madrid, Spain. ${ }^{7}$ Songshan Lake Materials Laboratory, Dongguan, 523808 Guangdong, China

\section{Conflict of interest}

The authors declare that they have no conflict of interest.

\section{Publisher's note}

Springer Nature remains neutral with regard to jurisdictional claims in published maps and institutional affiliations.

Supplementary information is available for this paper at https://doi.org/ 10.1038/s41427-019-0151-9.

Received: 18 May 2019 Revised: 25 June 2019 Accepted: 28 June 2019. Published online: 27 September 2019

\section{References}

1. Ramesh, R. \& Spaldin, N. A. Multiferroics: progress and prospects in thin films. Nat. Mater. 6, 21-29 (2007).

2. $\mathrm{Ma}, \mathrm{J}$., Hu, J., Li, Z. \& Nan, C. W. Recent progress in multiferroic magnetoelectric composites: from bulk to thin films. Adv. Mater. 23, 1062-1087 (2011).

3. Fusil, S., Garcia, V., Barthélémy, A. \& Bibes, M. Magnetoelectric devices for spintronics. Annu. Rev. Mater. Res. 44, 91-116 (2014).

4. Ortega, N., Kumar, A., Scott, J. F. \& Katiyar, R. S. Multifunctional magnetoelectric materials for device applications. J. Phys. Condens. Matter 27, 504002 (2015).

5. Dong, S., Liu, J.-M., Cheong, S.-W. \& Ren, Z. Multiferroic materials and magnetoelectric physics: symmetry, entanglement, excitation, and topology. Adv. Phys. 64, 519-626 (2015).

6. Fiebig, M. Revival of the magnetoelectric effect. J. Phys. D Appl. Phys. 38, R123-R152 (2005).

7. Rivera, J. P. A short review of the magnetoelectric effect and related experimental techniques on single phase (multi-) ferroics. Eur. Phys. J. B 71, 299-313 (2009).

8. Dzyaloshinskii, I. E. On the magneto-electrical effects in antiferromagnets. Sov. Phys. JETP 10, 628-629 (1960).

9. Astrov, D. N. The magnetoelectric effect in antiferromagnetics. Sov. Phys. JETP 11, 708-709 (1960).

10. Schmid, H. Multi-ferroic magnetoelectrics. Ferroelectrics 162, 317-338 (1994).

11. Kimura, T. et al. Magnetic control of ferroelectric polarization. Nature $\mathbf{4 2 6}$, $55-58(2003)$

12. Tokura, Y., Seki, S. \& Nagaosa, N. Multiferroics of spin origin. Rep. Prog. Phys. 77, 076501 (2014).

13. Wang, $X$. et al. Observation of magnetoelectric multiferroicity in a cubic perovskite system: $\mathrm{LaMn}_{3} \mathrm{Cr}_{4} \mathrm{O}_{12}$. Phys. Rev. Lett. 115, 087601 (2015).

14. Zhou, L. et al. Realization of large electric polarization and strong magnetoelectric coupling in $\mathrm{BiMn}_{3} \mathrm{Cr}_{4} \mathrm{O}_{12}$. Adv. Mater. 29, 1703435 (2017).

15. Eerenstein, W., Mathur, N. D. \& Scott, J. F. Multiferroic and magnetoelectric materials. Nature 442, 759-765 (2006).

16. Tokura, Y. \& Seki, S. Multiferroics with spiral spin orders. Adv. Mater. 22 1554-1565 (2010). 
17. Kim, J. Y., Koo, T. Y. \& Park, J. H. Orbital and bonding anisotropy in a half-filled $\mathrm{GaFeO}_{3}$ magnetoelectric ferrimagnet. Phys. Rev. Lett. 96, 047205 (2006).

18. Zhao, Z. Y. et al. Ground state and magnetic phase transitions of orthoferrite DyFeO . Phys. Rev. B 89, 224405 (2014).

19. Oh, Y. S. et al. Non-hysteretic colossal magnetoelectricity in a collinear antiferromagnet. Nat. Commun. 5, 3201 (2014).

20. Tezuka, K. \& Hinatsu, Y. Magnetic and crystallographic properties of $\mathrm{LnCrO}_{4}$ $(\mathrm{Ln}=\mathrm{Nd}, \mathrm{Sm}$, and Dy). J. Solid State Chem. 160, 362-367 (2001).

21. Steiner, M., Dachs, H. \& Ott, H. The determination of the magnetic structure of DyCrO 4 by neutron diffraction. Solid State Commun. 29, 231-234 (1979).

22. Midya, A., Khan, N., Bhoi, D. \& Mandal, P. 3d-4f spin interaction induced giant magnetocaloric effect in zircon-type $\mathrm{DyCrO}_{4}$ and $\mathrm{HoCrO}_{4}$ compounds. Appl. Phys. Lett. 103, 092402 (2013).

23. Long, Y. W. et al. Low-temperature neutron diffraction study of the crystal and magnetic phase transitions in $\mathrm{DyCrO}_{4}$. J. Magn. Magn. Mater. 322, 1912-1916 (2010).

24. Ray, A. \& Maitra, T. Electronic structure and $3 d-4 f$ exchange interactions in zircon-type $\mathrm{RCrO}_{4}$ oxides ( $\mathrm{R}=\mathrm{Dy}$, Ho and Gd). AIP Conf. Proc. 1665, 090017 (2015).

25. Long, Y. W. et al. High-pressure Raman scattering study on zircon-to scheelitetype structural phase transitions of $\mathrm{RCrO}_{4}$. J. Appl. Phys. 103, 093542 (2008).

26. Long, Y. W., Liu, Q. Q., Lv, Y. X., Yu, R. C. \& Jin, C. Q. Various 3d-4f spin interactions and field-induced metamagnetism in the $\mathrm{Cr}^{5+}$ system $\mathrm{DyCrO}$. Phys. Rev. B 83, 024416 (2011).

27. Ray, A. \& Maitra, T. Nature of transport gap and magnetic order in zircon and scheelite type $\mathrm{DyCrO}_{4}$ from first principles. J. Phys. Condens. Matter 27, 105501 (2015).

28. santos-García, A. J. D. et al. Determination of the crystal and magnetic structure of the $\mathrm{DyCrO}_{4}$-scheelite polymorph by neutron diffraction. J. Phys. Conf. Ser. 549, 012021 (2014).

29. Larson, A. C. \& Dreele, R. B. V. General Structure Analysis System (GSAS). Los Alamos National Laboratory Report (Los Alamos National Laboratory, Los Alamos, 2000).

30. Rodríguez-Carvajal, J. Recent advances in magnetic structure determination by neutron powder diffraction. Phys. B 192, 55-69 (1993)
31. Shtrikman, S. \& Treves, D. Observation of the magnetoelectric effect in $\mathrm{Cr}_{2} \mathrm{O}_{3}$ powders. Phys. Rev. 130, 986-988 (1963).

32. Liu, Y. Y., Xie, S. H., Jin, G. \& Li, J. Y. The effective magnetoelectric coefficients of polycrystalline $\mathrm{Cr}_{2} \mathrm{O}_{3}$ annealed in perpendicular electric and magnetic fields. J. Appl. Phys. 105, 073917 (2009).

33. Wiegelmann, H., Jansen, A. G. M., Wyder, P., Rivera, J. P. \& Schmid, H. Magnetoelectric effect of $\mathrm{Cr}_{2} \mathrm{O}_{3}$ in strong static magnetic fields. Ferroelectrics $\mathbf{1 6 2}$, 141-146 (1994).

34. Iyama, A. \& Kimura, T. Magnetoelectric hysteresis loops in $\mathrm{Cr}_{2} \mathrm{O}_{3}$ at room temperature. Phys. Rev. B 87, 180408 (2013).

35. Yin, L. H. et al. Colossal magnetodielectric effect and spin flop in magnetoelectric $\mathrm{CO}_{4} \mathrm{Nb}_{2} \mathrm{O}_{9}$ crystal. Appl. Phys. Lett. 109, 032905 (2016).

36. Rivera, J. P. On definitions, units, measurements, tensor forms of the linear magnetoelectric effect and on a new dynamic method applied to $\mathrm{Cr}-\mathrm{Cl}$ boracite. Ferroelectrics 161, 165-180 (1994).

37. Lee, N. et al. Giant tunability of ferroelectric polarization in $\mathrm{GdMn}_{2} \mathrm{O}_{5}$. Phys. Rev. Lett. 110, 137203 (2013)

38. Kimura, T., Lashley, J. C. \& Ramirez, A. P. Inversion-symmetry breaking in the noncollinear magnetic phase of the triangular-lattice antiferromagnet $\mathrm{CuFeO}_{2}$. Phys. Rev. B 73, 220401 (2006).

39. Kurumaji, T., Ishiwata, S. \& Tokura, Y. Doping-tunable ferrimagnetic phase with large linear magnetoelectric effect in a polar magnet $\mathrm{Fe}_{2} \mathrm{Mo}_{3} \mathrm{O}_{8}$. Phys. Rev. $X \mathbf{5}$, 031034 (2015).

40. Zhai, K. et al. Giant magnetoelectric effects achieved by tuning spin cone symmetry in Y-type hexaferrites. Nat. Commun. 8, 519 (2017).

41. Hur, $\mathrm{N}$. et al. Electric polarization reversal and memory in a multiferroic material induced by magnetic fields. Nature 429, 392-395 (2004).

42. Andreev, A. V., Bartashevich, M. I. \& Goto, T. Magnetostriction of UNiGa at metamagnetic transition. J. Alloy Compd. 219, 267-270 (1995).

43. Lee, J. H. et al. A strong ferroelectric ferromagnet created by means of spinlattice coupling. Nature 466, 954-958 (2010).

44. Tokunaga, $Y$. et al. Composite domain walls in a multiferroic perovskite ferrite. Nat. Mater. 8, 558-562 (2009).

45. Tokunaga, Y., Iguchi, S., Arima, T. \& Tokura, Y. Magnetic-field-induced ferroelectric state in $\mathrm{DyFeO}_{3}$. Phys. Rev. Lett. 101, 097205 (2008). 